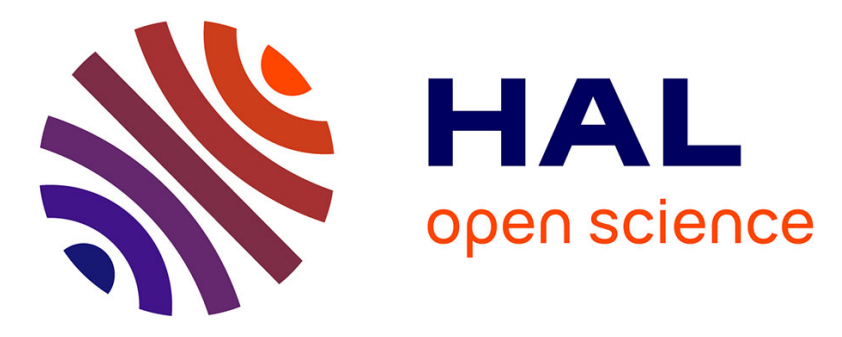

\title{
Hyperspectral anomaly detectors using robust estimators
}

\author{
Joana Frontera-Pons, Miguel Angel Veganzones, Frédéric Pascal, \\ Jean-Philippe Ovarlez
}

\section{- To cite this version:}

Joana Frontera-Pons, Miguel Angel Veganzones, Frédéric Pascal, Jean-Philippe Ovarlez. Hyperspectral anomaly detectors using robust estimators. IEEE Journal of Selected Topics in Applied Earth Observations and Remote Sensing, 2016, 9 (2), pp.720-731. 10.1109/JSTARS.2015.2453014 . hal01377668

\section{HAL Id: hal-01377668 https://hal.science/hal-01377668}

Submitted on 26 Feb 2020

HAL is a multi-disciplinary open access archive for the deposit and dissemination of scientific research documents, whether they are published or not. The documents may come from teaching and research institutions in France or abroad, or from public or private research centers.
L'archive ouverte pluridisciplinaire HAL, est destinée au dépôt et à la diffusion de documents scientifiques de niveau recherche, publiés ou non, émanant des établissements d'enseignement et de recherche français ou étrangers, des laboratoires publics ou privés. 


\title{
Hyperspectral Anomaly Detectors using Robust Estimators
}

\author{
Joana Frontera-Pons, Student Member, IEEE, Miguel A. Veganzones, Member, IEEE, Frédéric Pascal, Senior \\ Member, IEEE, and Jean-Philippe Ovarlez, Member, IEEE
}

\begin{abstract}
Anomaly detection methods are devoted to target detection schemes in which no a priori information about the spectra of the targets of interest is available. This paper reviews classical anomaly detection schemes such as the widely spread Reed-Xiaoli Detector and some of its variations. Moreover, the Mahalanobis distance based detector, rigorously derived from a Kelly's test-based approach, is analyzed and its exact distribution is derived when both mean vector and covariance matrix are unknown and have to be estimated. Although, most of these techniques are based on Gaussian distribution, we also propose here ways to extend them to non-Gaussian framework. For this purpose, elliptical distributions are considered for background statistical characterization. Through this assumption, this paper describes robust estimation procedures (M-estimators of location and scale) more suitable for non-Gaussian environment. We show that using them as plug-in estimators in anomaly detectors leads to some great improvement in the detection process. Finally, the theoretical contribution is validated through simulations and on real hyperspectral scenes.
\end{abstract}

Index Terms-Hyperspectral Imaging, anomaly detection, elliptical distributions, $M$-estimation.

\section{INTRODUCTION}

$\mathbf{T}$ ARGET detection and anomaly detection of multidimensional signals have proved to be valuable techniques in a wide range of applications, including search-and-rescue, surveillance, rare mineral and land mines detection, etc (see for e.g. [1], [2], [3]). Target detection aims to discover the presence of a specific signal of interest among a set of signals. Statistical target detection is based on the Neyman-Pearson (NP) criterion, which maximizes the probability of detection (PD) for a given probability of false alarm (PFA).

Classical target detection methods require the knowledge of the spectra of the desired targets. One could be interested in a large number of possible targets each with different signatures. Thus, the variety of sought spectra corresponding to the different kind of targets and the difficulties due to the atmospheric compensation for the measured spectral signatures (used as steering vectors) have led to the derivation

\footnotetext{
J. Frontera-Pons is with SONDRA, Supelec, Plateau du Moulon, 3 rue Joliot-Curie, F-91190 Gif-sur-Yvette, France (e-mail: joana.fronterapons@supelec.fr)

M. A Veganzones is with GIPSA-lab, CNRS, 11 rue des mathmatiques, F38402 Saint Martin d'Hres , France (e-mail: miguel-angel.veganzones@gipsalab.fr)

F. Pascal is with SONDRA, Supelec, Plateau du Moulon, 3 rue Joliot-Curie, F-91190 Gif-sur-Yvette, France (e-mail: frederic.pascal@supelec.fr)

J.-P. Ovarlez is with ONERA, DEMR/TSI, Chemin de la Hunière, F-91120 Palaiseau, France (e-mail: jean-philippe.ovarlez@onera.fr)
}

of new algorithms that intend to distinguish unusual materials in a scene without reference to target signatures. In this work, we are focused on anomaly detection (see e.g. [4] and references therein). It can be interpreted as a particular case of target detection in which no a priori information about the spectra of the targets of interest is available. Hence, the aim of anomaly detection is to locate objects in the image that are anomalous with respect to the background. The type of interesting targets can differ significantly from one application to another, e.g. in forestry applications infected trees are the anomalies of interest, whereas in defense and intelligence applications, the anomalies to be detected are usually vehicles. Note that, since anomaly detectors do not use any a priori knowledge, they cannot distinguish between true targets and detections of bright pixels of the background or targets that are not of interest. This fact makes extremely difficult to define a false alarm rate for the detectors as highlighted in [5].

Anomalies are defined with reference to a model of the background. As for the previous target detection methods, the background model is developed adaptively using reference data (see e.g. [6] for a complete survey in anomaly detection methods). Most of these methods rely on the classical Gaussian distribution assumption and need for the statistical characterization of the background usually through first and second order parameters (i.e. the mean vector and the covariance matrix). In this case, the reference data are taken either from a local neighborhood around the observation vector either using all the pixels in the image. Both approaches have their benefits (see e.g. [7]). Local strategy provides more realistic scenario for the background characterization. However, it can be sensitive to the presence of false alarms due to isolated anomalies. While the global approach is not likely to generate this kind of false alarms, it will decrease the detection capability for isolated targets. From here on, local procedures will be considered for the different detection schemes.

\section{A. Related work}

The actual distribution of the background pixels differs from the theoretically predicted under Gaussian hypothesis (see [8] for a recent overview on background modelling for HSI). In fact, as stated in [9], [10], the empirical distribution usually has heavier tails compared to the Gaussian distribution, and these tails strongly influence the observed false-alarm rate of the detector. Therefore, the class of Elliptical distributions 
is assumed for background statistics characterization. The family of Elliptical distributions were originally introduced by Kelker in [11] and widely studied in [12]. They account for non-Gaussianity providing a long tailed alternative to multivariate normal model. They are proven to represent a more accurate characterization of HSI than models based on Gaussian assumption [9]. However, when considering elliptical distributions, the classical Gaussian-based estimators do not provide optimal performance anymore. Complementary to the paper [8] focused on the non-Gaussian background modeling with Elliptical distributions, this work aims at studying appropriate robust parameters estimates.

\section{B. Contributions}

We consider on the first part of the paper the most popular Gaussian-based anomaly detectors, and we provide a rigorous derivation of the Mahalanobis distance through a Kelly's testbased approach. Moreover, one of the theoretical contribution is the derivation of the exact distribution for the classical Mahalanobis-based anomaly detector when both the mean vector and the covariance matrix are unknown in Gaussian environment.

Furthermore, robust estimation methods are considered in classical anomaly detection schemes for non-Gaussian distributed background assumption, and the improvement brought in most scenarios is pointed out. The Fixed Point estimators (also known as Tyler's estimators [13]) are proposed for the parameters estimation. These can then be used as plug-in estimators in place of the unknown mean vector or/and of the covariance matrix in the detection scheme (see for e.g. [14], [15]). This is a simple but often efficient method to obtain robust properties for signal processors derived under the Gaussian assumption. One of the contributions of this work is to extend the results presented in [16].

\section{Outline and notation}

This paper is organized as follows. Section II revisits classical anomaly detection schemes and provides the theoretical contribution of this paper by deriving the distribution of one of the detectors. Section III describes the family of elliptical distributions and the robust estimation methods studied in this paper for anomaly detection purposes. Section IV illustrates the theoretical analysis through simulations and Section $\mathrm{V}$ reveals the theoretical improvement over real hyperspectral images. Finally, Section VI concludes this work.

In the following, vectors (resp. matrices) are denoted by bold-faced lowercase letters (resp. uppercase letters). ${ }^{T}$ represents the transpose operator. $|\mathbf{A}|$ represents the determinant of the matrix $\mathbf{A}$ and $\operatorname{Tr}(\mathbf{A})$ its trace. $j$ is used to denote the unit imaginary number. $\sim$ means "distributed as". $\Gamma(\cdot)$ denotes the gamma function. Eventually, $\|\mathbf{x}\|$ represents the Euclidean norm of the vector $\mathbf{x}$.

\section{Adaptive Anomaly Detection methods}

Before detailing the analysis of the corresponding detectors, let us recap the most common Gaussian-based estimators.
Along with their well-known properties and their simplicity of analysis, the Sample Covariance Matrix (SCM) and the Sample Mean Vector (SMV) are the most extended estimates since they are the Maximum Likelihood Estimators (MLE) for Gaussian case, as shown in [17]:

$$
\begin{aligned}
\hat{\boldsymbol{\mu}}_{S M V} & =\frac{1}{N} \sum_{i=1}^{N} \mathbf{x}_{i} \\
\hat{\boldsymbol{\Sigma}}_{S C M} & =\frac{1}{N} \sum_{i=1}^{N}\left(\mathbf{x}_{i}-\hat{\boldsymbol{\mu}}_{S M V}\right)\left(\mathbf{x}_{i}-\hat{\boldsymbol{\mu}}_{S M V}\right)^{T} .
\end{aligned}
$$

Further, we shall denote the Centered SCM (CSCM) as:

$$
\hat{\boldsymbol{\Sigma}}_{C S C M}=\frac{1}{N} \sum_{i=1}^{N}\left(\mathbf{x}_{i}-\boldsymbol{\mu}\right)\left(\mathbf{x}_{i}-\boldsymbol{\mu}\right)^{T} .
$$

where $N$ denotes the number of secondary data. However, such widespread techniques are suboptimal when the noise is a non-Gaussian stochastic process. Section III reviews some robust procedures particularly suited for estimating the covariance matrix and the mean vector of elliptical populations. Let us now detail the most popular Gaussian-based anomaly detectors .

\section{A. Reed-Xiaoli Detector}

The original Reed-Xiaoli Detector (RXD) proposed in [18] is commonly considered as the benchmark anomaly detector for hyperspectral data. The considered signal model can be written as:

$$
\begin{cases}\mathcal{H}_{0}: \mathbf{x}_{i}=\mathbf{b}_{i}, & , i=1, \ldots, N \\ \mathcal{H}_{1}: \mathbf{x}_{i}=\mathbf{p} \alpha_{i}+\mathbf{b}_{i}, & , i=1, \ldots, N,\end{cases}
$$

where $\mathbf{x}_{i}$ are the $N$ available data vectors on the image of dimension $m . \mathbf{b}_{i} \sim \mathcal{N}(\mathbf{0}, \boldsymbol{\Sigma})$ represents the residual background, $\mathbf{p}$ is the spectral signature of the possible anomalous material assumed to be unknown; and $\alpha_{i}$ stands for the amplitude of the intended targets through the $N$ available data, i.e. it is a known vector $\boldsymbol{\alpha}$ of dimension $N$ that indicates the strength and position of the sought targets over the image. Remark that each vector from the available data can potentially contain an anomaly while in classical detection problem secondary data are assumed to be signal free. Thus, one can arrange the vector data into a matrix as $\mathbf{X}=\left[\mathbf{x}_{1}, \mathbf{x}_{2}, \ldots, \mathbf{x}_{N}\right]$, and the detection scheme derived in [18] takes the form:

$$
\Lambda(\mathbf{X})=\frac{\left(\mathbf{X} \boldsymbol{\alpha}^{T}\right)^{T}\left(\mathbf{X} \mathbf{X}^{T}\right)^{-1}\left(\mathbf{X} \boldsymbol{\alpha}^{T}\right)}{\boldsymbol{\alpha} \boldsymbol{\alpha}^{T}} \underset{\mathcal{H}_{0}}{\stackrel{\mathcal{H}_{1}}{\gtrless}} \lambda .
$$

Since hyperspectral data are not zero mean, let us now consider that the background $\mathbf{b}_{i}$ is distributed according to $\mathcal{N}(\boldsymbol{\mu}, \boldsymbol{\Sigma})$ and the mean vector $\boldsymbol{\mu}$ is supposed to be known. In the case just one anomaly in the data under test is intended to be detected, the corresponding amplitude vector can be written as $\boldsymbol{\alpha}_{i}=\left[\begin{array}{llllll}0 & \ldots & 0 & 1 & 0 & \ldots\end{array}\right]^{T}$ where 1 is at the $\mathrm{i}^{\text {th }}$ position and the previous detector, whatever $i \in[1, N]$, takes the form:

$$
\Lambda_{R X D}=\left(\mathbf{x}_{i}-\boldsymbol{\mu}\right)^{T} \hat{\boldsymbol{\Sigma}}_{C S C M}^{-1}\left(\mathbf{x}_{i}-\boldsymbol{\mu}\right) \underset{\mathcal{H}_{0}}{\stackrel{\mathcal{H}_{1}}{\gtrless}} \lambda .
$$


Finally, since the mean vector is usually unknown, it can be replaced on the detector in by its estimate $\hat{\boldsymbol{\mu}}_{S M V}$. The resulting detector, commonly known as two-step Generalized Likelihood Ratio Test, yields:

$$
\Lambda_{A R X D}=\left(\mathbf{x}_{i}-\hat{\boldsymbol{\mu}}_{S M V}\right)^{T} \hat{\boldsymbol{\Sigma}}_{S C M}^{-1}\left(\mathbf{x}_{i}-\hat{\boldsymbol{\mu}}_{S M V}\right) \underset{\mathcal{H}_{0}}{\stackrel{\mathcal{H}_{1}}{\gtrless}} \lambda .
$$

The covariance matrix estimation $\hat{\boldsymbol{\Sigma}}_{S C M}$ in Eq. (4), is performed over all the data set, i.e. including the vector $\mathbf{x}_{i}$ under test. In the following, the test in Eq. (4) will be referred as the Adaptive RXD (ARXD), to underline the fact that the unknown mean vector is replaced by its estimate.

\section{B. Kelly Anomaly Detector}

We detail here a classical anomaly detector often mistakenly referred as the RXD. Following the development proposed in [19], let us now assume the following signal model:

$$
\begin{cases}\mathcal{H}_{0}: \mathbf{x}=\mathbf{b}, & \mathbf{x}_{i}=\mathbf{b}_{i}, i=1, \ldots, N \\ \mathcal{H}_{1}: \mathbf{x}=\alpha \mathbf{p}+\mathbf{b}, & \mathbf{x}_{i}=\mathbf{b}_{i}, i=1, \ldots, N\end{cases}
$$

and the $\mathbf{b}_{1}, \ldots \mathbf{b}_{N}$ are assumed to an independent identically distributed (IID) sample from a Gaussian distribution $\mathbf{b}_{i} \sim$ $\mathcal{N}(\boldsymbol{\mu}, \boldsymbol{\Sigma})$ As in classical Kelly detector, the covariance matrix $\boldsymbol{\Sigma}$ is unknown and the mean vector $\boldsymbol{\mu}$ is supposed to be known. However for anomaly detector derivation, the amplitude of the signal $\alpha$ is supposed to be known and the unknown parameter is now the steering vector $\mathbf{p}$. Therefore, $N+1 \mathrm{~m}$-dimensional vectors are observed under each hypothesis and the joint probability density function (p.d.f.) of the the $N$ secondary data and the observation vector $\mathbf{x}$ under the two hypotheses $\mathcal{H}_{i}$ can be written as:

$$
f_{i}(\mathbf{x})=\left(\frac{1}{2 \pi^{\frac{m}{2}}|\boldsymbol{\Sigma}|^{\frac{1}{2}}} \exp \left[-\frac{1}{2} \operatorname{Tr}\left(\boldsymbol{\Sigma}^{-1} \mathbf{T}_{i}\right)\right]\right)^{N+1}
$$

where $\mathbf{T}_{i}$ is the composite sample covariance matrix constructed from both the secondary data and observation vector:

$$
\begin{aligned}
\mathbf{T}_{0} & =\frac{1}{N+1}\left((\mathbf{x}-\boldsymbol{\mu})(\mathbf{x}-\boldsymbol{\mu})^{T}+\hat{\mathbf{W}}\right), \\
\mathbf{T}_{1} & =\frac{1}{N+1}\left((\mathbf{x}-(\alpha \mathbf{p}+\boldsymbol{\mu}))(\mathbf{x}-(\alpha \mathbf{p}+\boldsymbol{\mu}))^{T}+\hat{\mathbf{W}}\right),
\end{aligned}
$$

and $\hat{\mathbf{W}}=N \hat{\boldsymbol{\Sigma}}_{C S C M}$. The first step is to maximize with respect to (w.r.t) the unknown covariance matrix $\boldsymbol{\Sigma}$. Thus, the matrix maximizing the $\operatorname{PDF} f_{i}$ is simply $\mathbf{T}_{i}$. When this estimator is replaced in the PDF, one obtains:

$$
\max _{\Sigma} f_{i}=\left(\frac{1}{(\pi e)^{m}\left|\mathbf{T}_{i}\right|}\right)^{\frac{N+1}{2}} .
$$

and the Generalized Likelihood Ratio Test (GLRT), neglecting the exponent $(N+1) / 2$ is given by:

$$
\Lambda(\mathbf{x}, \mathbf{p})=\frac{\left|\mathbf{T}_{0}\right|}{\left|\mathbf{T}_{1}\right|} \underset{\mathcal{H}_{0}}{\stackrel{\mathcal{H}_{1}}{\gtrless}} \eta
$$

It remains to maximize this expression over the unknown spectral signature $\mathbf{p}$ and the resulting MLE takes the form:

$$
\hat{\mathbf{p}}=\frac{\mathbf{x}-\boldsymbol{\mu}}{\alpha} .
$$

After replacing p by Eq. (8) in Eq. (7), it is easy to show that the resulting GLRT test is equivalent to:

$$
\Lambda_{\text {KellyAD } \hat{\boldsymbol{\Sigma}}}=(\mathbf{x}-\boldsymbol{\mu})^{T} \hat{\boldsymbol{\Sigma}}_{C S C M}^{-1}(\mathbf{x}-\boldsymbol{\mu}) \underset{\mathcal{H}_{0}}{\stackrel{\mathcal{H}_{1}}{\gtrless}} \lambda .
$$

The quadratic form in Eq. (9) corresponds to the Mahalanobis distance detailed in [20]. It performs statistically as an outlier detector. When Gaussian assumption is valid, the quadratic form $(\mathbf{x}-\boldsymbol{\mu})^{T} \boldsymbol{\Sigma}^{-1}(\mathbf{x}-\boldsymbol{\mu})$ follows a $\chi^{2}$-distribution with $m$ degrees of freedom for $\boldsymbol{\Sigma}$ and $\boldsymbol{\mu}$ perfectly known. In case the parameter $\Sigma$ is replaced by its MLE, the CSCM, the distribution of the quadratic form can be written according to (see [21]):

$$
\Lambda_{\text {KellyAD } \hat{\boldsymbol{\Sigma}}}^{(N)} \sim T^{2}
$$

becomes a Hotelling $T^{2}$ distribution and thus,

$$
\frac{N-m+1}{m N} \Lambda_{\text {KellyAD } \hat{\boldsymbol{\Sigma}}}^{(N)} \sim F_{m, N-m+1}
$$

where $F_{m, N-m+1}$ is the non-central $F$-distribution with $m$ and $N-m+1$ degrees of freedom [22] and the superscript $(N)$ is used to stress the dependence on the number of secondary data $N$. For high values of $N,(N>10 \mathrm{~m})$, the distribution can be approximated by the $\chi^{2}$-distribution.

As discussed above, when the mean vector is unknown, it can be replaced on the detector (two-step GLRT) by its MLE leading to:

$$
\Lambda_{K e l l y A D \hat{\boldsymbol{\Sigma}}, \hat{\boldsymbol{\mu}}}^{(N)}=\left(\mathbf{x}-\hat{\boldsymbol{\mu}}_{S M V}\right)^{T} \hat{\boldsymbol{\Sigma}}_{S C M}^{-1}\left(\mathbf{x}-\hat{\boldsymbol{\mu}}_{S M V}\right) \underset{\mathcal{H}_{0}}{\stackrel{\mathcal{H}_{1}}{\gtrless}} \lambda .
$$

Remark II.1. Interestingly, note that $\Lambda_{R X D}\left(\right.$ resp. $\left.\Lambda_{A R X D}\right)$ and the $\Lambda_{\text {KellyAD } \hat{\boldsymbol{\Sigma}}}$ (reps. $\Lambda_{\text {KellyAD } \hat{\boldsymbol{\Sigma}}, \hat{\boldsymbol{\mu}}}^{(N)}$ ) differ only on the fact that the vector $\mathrm{x}$ under test is also present in the covariance matrix estimation in Eq. (4). Therefore, in $\Lambda_{R X D}$, the $N$ secondary data are not assumed to be signal free and the proposed detector aims to compare every sample to the covariance matrix over all the samples. While in the second approach, $\Lambda_{\text {KellyAD } \hat{\mathbf{\Sigma}}}$, one intends to differentiate the observation vector from the background statistically characterized using $N$ samples. Hence, $N+1$ vectors are available in the latter and $\Lambda_{\text {KellyAD } \hat{\Sigma}}$ does not represent anymore a benchmark structure. Often, the local Kelly detector is mistakenly referred as the local RXD when the users, either remove the vector $\mathbf{x}_{i}$ from the secondary data or they prevent it to be part of this set by using a guard window.

The distribution of this detection test is given in the next Proposition. 
Proposition II.1. The distribution of the detector under Gaussian assumption is given by

$$
\frac{N-m}{m(N+1)} \Lambda_{\text {KellyAD } \hat{\boldsymbol{\Sigma}}, \hat{\boldsymbol{\mu}}}^{(N)} \sim F_{m, N-m},
$$

with $F_{m, N-m}$ is the non-central $F$-distribution with $m$ and $N-m$ degrees of freedom.

Proof: For simplicity matters, the following notations are used: $\hat{\boldsymbol{\Sigma}}=\hat{\boldsymbol{\Sigma}}_{S C M}$ and $\hat{\boldsymbol{\mu}}=\hat{\boldsymbol{\mu}}_{S M V}$.

Let us set $\forall i=1, \ldots, N, \mathbf{x}_{i} \sim \mathcal{N}(\boldsymbol{\mu}, \boldsymbol{\Sigma})$ and $\mathbf{x} \sim \mathcal{N}(\boldsymbol{\mu}, \boldsymbol{\Sigma})$, where all these vectors are independent. Now, let us denote

$$
\hat{\mathbf{W}}_{N-1}=\sum_{i=1}^{N}\left(\mathbf{x}_{i}-\hat{\boldsymbol{\mu}}\right)\left(\mathbf{x}_{i}-\hat{\boldsymbol{\mu}}\right)^{T}=N \hat{\boldsymbol{\Sigma}}_{S C M} .
$$

Note that as an application of the Cochran theorem (see e.g. [23]), one has

$$
\hat{\mathbf{W}}_{N-1} \stackrel{\text { dist. }}{=} \sum_{i=1}^{N-1}\left(\mathbf{x}_{i}-\boldsymbol{\mu}\right)\left(\mathbf{x}_{i}-\boldsymbol{\mu}\right)^{T}=(N-1) \hat{\boldsymbol{\Sigma}}_{C S C M},
$$

where $\stackrel{\text { dist. }}{=}$ means is distributed as.

Since $\hat{\boldsymbol{\mu}} \sim \mathcal{N}\left(\boldsymbol{\mu}, \frac{1}{N} \boldsymbol{\Sigma}\right)$, one has $\mathbf{x}-\hat{\boldsymbol{\mu}} \sim \mathcal{N}\left(\mathbf{0}, \frac{N+1}{N} \boldsymbol{\Sigma}\right)$. This can be equivalently rewritten as

$$
\mathbf{y}=\sqrt{N /(N+1)}(\mathbf{x}-\hat{\boldsymbol{\mu}}) \sim \mathcal{N}(\mathbf{0}, \boldsymbol{\Sigma}) .
$$

As we jointly estimate the mean and the covariance matrix, a degree of freedom is lost, compared with the only covariance matrix estimation problem.

Let us now consider $\Lambda_{\text {KellyAD } \hat{\boldsymbol{\Sigma}}}^{(N-1)}$ (i.e. $\boldsymbol{\mu}$ known) built from $N-1$ secondary data, rewritten in terms of $\hat{\mathbf{W}}_{N-1}$ :

$$
\Lambda_{\text {KellyAD } \hat{\boldsymbol{\Sigma}}}^{(N-1)}=(N-1)\left((\mathbf{x}-\boldsymbol{\mu})^{T} \hat{\mathbf{W}}_{N-1}^{-1}(\mathbf{x}-\boldsymbol{\mu})\right)
$$

where $(\mathbf{x}-\boldsymbol{\mu}) \sim \mathcal{N}(\mathbf{0}, \boldsymbol{\Sigma})$ and whose distribution is given by Eq. (11) where $N$ is replaced by $N-1$.

Now, for the joint estimation problem, the $\Lambda_{K e l l y A D \hat{\boldsymbol{\Sigma}}, \hat{\boldsymbol{\mu}}}$ can be rewritten as:

$$
\begin{aligned}
\Lambda_{\text {KellyAD } \hat{\boldsymbol{\Sigma}}, \hat{\boldsymbol{\mu}}}^{(N)} & =N\left((\mathbf{x}-\hat{\boldsymbol{\mu}})^{T} \hat{\mathbf{W}}_{N-1}^{-1}(\mathbf{x}-\hat{\boldsymbol{\mu}})\right) \\
& =N \frac{N+1}{N}\left(\mathbf{y}^{T} \hat{\mathbf{W}}_{N-1}^{-1} \mathbf{y}\right) \\
& \stackrel{\text { dist. }}{=} \frac{N+1}{N-1} \Lambda_{\text {KellyAD } \hat{\boldsymbol{\Sigma}}}^{(N-1)}
\end{aligned}
$$

This concludes the proof.

The "PFA-threshold" relationship is easily obtained as the complementary cumulative density function (c.d.f.) of the detector distribution.

It is worth pointing out from Eq. (12) that $\Lambda_{\text {KellyAD } \hat{\boldsymbol{\Sigma}}, \hat{\boldsymbol{\mu}}}$ performs similarly to a matched filter structure applied to $\mathbf{x}-\hat{\boldsymbol{\mu}}_{S M V}:$

$$
\Lambda(\mathbf{x})=c \mathbf{H}^{T}\left(\mathbf{x}-\hat{\boldsymbol{\mu}}_{S M V}\right),
$$

where $\mathbf{H}^{T}$ is the matched signal and $c$ a constant that can be also a function on $\mathbf{x}$. The expression in Eq. (14) is completely characterized by the matched signal $\mathbf{H}^{T}$ and the scale constant $c$. Hence, one can identify from Eq. (12) the matched signal $\mathbf{H}^{T}=\left(\mathbf{x}-\hat{\boldsymbol{\mu}}_{S M V}\right)^{T} \hat{\boldsymbol{\Sigma}}_{S C M}^{-1}$ and $c=1$.

\section{Normalized-RXD and Uniform Target Detector}

Following the same approach than in Eq. (14), one can derive many different anomaly detection schemes. We recall here two popular variants of the Mahalanobis distance described in [4]: the Normalized-RXD (N-RXD) and the Uniform Target Detector (UTD).

The N-RXD takes the form:

$$
\Lambda_{N-R X D}=\frac{\left(\mathbf{x}-\hat{\boldsymbol{\mu}}_{S M V}\right)^{T}}{\left\|\mathbf{x}-\hat{\boldsymbol{\mu}}_{S M V}\right\|} \hat{\boldsymbol{\Sigma}}_{S C M}^{-1} \frac{\left(\mathbf{x}-\hat{\boldsymbol{\mu}}_{S M V}\right)}{\left\|\mathbf{x}-\hat{\boldsymbol{\mu}}_{S M V}\right\|} \underset{\mathcal{H}_{0}}{\stackrel{\mathcal{H}_{1}}{\gtrless}} \lambda,
$$

where $\left\|\mathbf{x}-\hat{\boldsymbol{\mu}}_{S M V}\right\|^{2}=\left(\mathbf{x}-\hat{\boldsymbol{\mu}}_{S M V}\right)^{T}\left(\mathbf{x}-\hat{\boldsymbol{\mu}}_{S M V}\right)$ stands for the Euclidean norm of the vector. The detection test in Eq. (15) can be immediately identified as the normalized version of $\Lambda_{\text {Kelly } A D}$. In addition, $\Lambda_{N-R X D}$ takes also the form of a matched filter specified in Eq. (14) with matched signal $\mathbf{H}^{T}=\left(\mathbf{x}-\hat{\boldsymbol{\mu}}_{S M V}\right)^{T} \hat{\boldsymbol{\Sigma}}_{S C M}^{-1}$ the same as in Eq. (9) and a different scale constant $c=\left\|\mathbf{x}-\hat{\boldsymbol{\mu}}_{S M V}\right\|^{-2}$.

The UTD is another widespread anomaly detection test. It was firstly introduced in [24] and can be defined as:

$$
\Lambda_{U T D}=\left(\mathbf{1}-\hat{\boldsymbol{\mu}}_{S M V}\right)^{T} \hat{\boldsymbol{\Sigma}}_{S C M}^{-1}\left(\mathbf{x}-\hat{\boldsymbol{\mu}}_{S M V}\right) \underset{\mathcal{H}_{0}}{\stackrel{\mathcal{H}_{1}}{\gtrless}} \lambda .
$$

with $\mathbf{1}=[1, \ldots, 1]^{T}$ is the $m$-dimensional unity vector. Once again the detector in Eq. (16) can be interpreted as a matched filter where $\mathbf{H}^{T}=\left(\mathbf{1}-\hat{\boldsymbol{\mu}}_{S M V}\right)^{T} \hat{\boldsymbol{\Sigma}}_{S C M}^{-1}$ is the matched signal. If there is no a priori information about the target spectra, the non-prior approach is the one that does not introduce any information into the detector and consists on assuming uniform distribution for the spectra over all the bands.

\section{Generalized Kelly Anomaly Detector}

In the previous detection schemes, it has not been taken into account in the derivation of the test that both mean vector $\boldsymbol{\mu}$ and covariance matrix $\boldsymbol{\Sigma}$ were unknown. One simply replaced the mean vector by a plug-in estimate in the detector (two-step GLRT). In case both covariance matrix and mean vector are unknown, we need to derive a new detector. This strategy is similar to the one proposed in [25] for the generalized Kelly detection test. The likelihood functions under $\mathcal{H}_{0}$ and $\mathcal{H}_{1}$ are given in (5). Under $\mathcal{H}_{0}$ and $\mathcal{H}_{1}$, the maxima are achieved at

$$
\max _{\boldsymbol{\Sigma}, \boldsymbol{\mu}} f_{i}=\left(\frac{1}{(\pi e)^{m}\left|\mathbf{T}_{i}\right|}\right)^{\frac{N+1}{2}}, \text { for } i=0,1,
$$

where

$$
\begin{gathered}
(N+1) \mathbf{T}_{0}=\left(\mathbf{x}-\boldsymbol{\mu}_{0}\right)\left(\mathbf{x}-\boldsymbol{\mu}_{0}\right)^{T}+\sum_{i=1}^{N}\left(\mathbf{x}_{i}-\boldsymbol{\mu}_{0}\right)\left(\mathbf{x}_{i}-\boldsymbol{\mu}_{0}\right)^{T}, \\
(N+1) \mathbf{T}_{1}=\left(\mathbf{x}-\alpha \mathbf{p}-\boldsymbol{\mu}_{1}\right)\left(\mathbf{x}-\alpha \mathbf{p}-\boldsymbol{\mu}_{1}\right)^{T} \\
+\sum_{i=1}^{N}\left(\mathbf{x}_{i}-\boldsymbol{\mu}_{1}\right)\left(\mathbf{x}_{i}-\boldsymbol{\mu}_{1}\right)^{T},
\end{gathered}
$$


and

$$
\begin{aligned}
& \boldsymbol{\mu}_{0}=\frac{1}{N+1}\left(\mathbf{x}+\sum_{i=1}^{N} \mathbf{x}_{i}\right) \\
& \boldsymbol{\mu}_{1}=\frac{1}{N+1}\left(\mathbf{x}-\alpha \mathbf{p}+\sum_{i=1}^{N} \mathbf{x}_{i}\right) .
\end{aligned}
$$

Following the same lines than in [25], one has to maximize the LR in Eq. (7) w.r.t. p. This is obtained by taking:

$$
\hat{\mathbf{p}}=\frac{N+1}{N} \frac{\left(\mathbf{x}-\boldsymbol{\mu}_{0}\right)}{\alpha} .
$$

Hence, the resulting detector can be written according to:

$$
\Lambda_{G-\text { KellyAD }}=\left(\mathbf{x}-\boldsymbol{\mu}_{0}\right)^{H} \mathbf{S}_{0}^{-1}\left(\mathbf{x}-\boldsymbol{\mu}_{0}\right) \underset{\mathcal{H}_{0}}{\stackrel{\mathcal{H}_{1}}{\gtrless}} \lambda,
$$

where $\mathbf{S}_{0}=\sum_{i=1}^{N}\left(\mathbf{x}_{i}-\boldsymbol{\mu}_{0}\right)\left(\mathbf{x}_{i}-\boldsymbol{\mu}_{0}\right)^{H}$, and $\boldsymbol{\mu}_{0}=$ $\frac{1}{N+1}\left(\mathbf{x}+\sum_{i=1}^{N} \mathbf{x}_{i}\right)$. Once again the mean vector estimate $\boldsymbol{\mu}_{0}$ and the covariance matrix $\mathbf{S}_{0}$ depend on the data under test $\mathbf{x}$. Hence, $\mathbf{x}-\boldsymbol{\mu}_{0}$ and $\mathbf{S}_{0}$ are not independent. Remark that one can write $\left(\mathbf{x}-\boldsymbol{\mu}_{0}\right)=\frac{N}{N+1}\left(\mathbf{x}-\hat{\boldsymbol{\mu}}_{S M V}\right)$. Neglecting the multiplicative constants, the test in Eq. (20) appears to be equivalent to the classical $\Lambda_{R X D}$ obtained throughout a different approach but built with $N+1$ available data.

\section{Robust Anomaly Detection}

In this section, the class of elliptical distributions and robust estimation procedures are reviewed.

\section{A. Elliptical Distributions}

Hyperspectral data have been proven not to be multivariate normal but long tailed distributed [9]. In order to take into account these features, the class of elliptical distributions (see for e.g. [12] and [26] for a complete survey on elliptical distributions) is considered to describe the statistical behavior of the hyperspectral background. An $m$-dimensional random real vector $\mathbf{x}$ has a multivariate elliptical distribution if its characteristic function is of the form:

$$
\Phi_{\mathbf{x}}(\mathbf{c})=\exp \left(j \mathbf{c}^{T} \boldsymbol{\mu}\right) \phi\left(\frac{1}{2} \mathbf{c}^{T} \boldsymbol{\Sigma} \mathbf{c}\right),
$$

for some function $\phi: \mathbb{R}^{+} \rightarrow \mathbb{R}$, called characteristic generator, a positive semidefinite matrix $\boldsymbol{\Sigma}$, called scatter matrix and $\boldsymbol{\mu} \in \mathbb{C}^{m}$ the location vector. We shall write $\mathbf{x} \sim \mathcal{E}(\boldsymbol{\mu}, \boldsymbol{\Sigma}, \phi)$. From Eq. (21), it does not follow that $\mathbf{x}$ has a p.d.f. $f_{\mathbf{x}}(\cdot)$, but if exists, it has the form:

$$
f_{\mathbf{x}}(\mathbf{x})=c_{m, h}|\boldsymbol{\Sigma}|^{-\frac{1}{2}} h_{m}\left(\frac{1}{2}(\mathbf{x}-\boldsymbol{\mu})^{T} \boldsymbol{\Sigma}^{-1}(\mathbf{x}-\boldsymbol{\mu})\right),
$$

where $c_{m, h}$ is a normalization constant and $h_{m}(\cdot)$ is any function such as Eq. (22) defines a p.d.f. in $\mathbb{R}^{m}$. The function $h_{m}$ is usually called density generator and it is assumed to be only approximately known. In this case, we shall write
$\mathcal{E}\left(\boldsymbol{\mu}, \boldsymbol{\Sigma}, h_{m}\right)$ instead of $\mathcal{E}(\boldsymbol{\mu}, \boldsymbol{\Sigma}, \phi)$. Remark that the p.d.f. in Eq. (22) depends on $\mathbf{x}$ only through the quadratic form $(\mathbf{x}-\boldsymbol{\mu})^{T} \boldsymbol{\Sigma}^{-1}(\mathbf{x}-\boldsymbol{\mu})$. Thus, the level sets of the density $f_{\mathbf{x}}(\mathbf{x})$ are ellipsoids in the Euclidean $m$-space.

If the second-order moment exists, then $\boldsymbol{\Sigma}$ reflects the structure of the covariance matrix of the elliptically distributed random vector $\mathbf{x}$, i.e. the covariance matrix is equal to the scatter matrix up to a scalar constant. It serves to characterize the correlation structure existing within the spectral bands. It is worth pointing out that the family of elliptical distributions includes a large number of distributions, notably the Gaussian distribution, multivariate $t$-distribution, $K$-distribution or multivariate Cauchy. Thus, it allows for heterogeneity of the background power with the texture.

In order to improve the parameter estimation, the objective is to find an appropriate model and to use the corresponding MLEs. Therefore, if the density generator $h_{m}$ is perfectly known, once could obtain the optimal MLEs for such $h_{m}$. This method leads to asymptotically efficient estimators but not necessarily robust. Indeed, the robust estimator is rather one that is still fairly reliable, regardless of the data departure, failing to be optimal in some scenarios. In a real life applications, although elliptical distributions offer a great deal of possible distributions, the risk that the data do not follow the model considered still remains. Thus, the models used always correspond to simplifications of the reality. The fact that a slight deviation between reality and the model assumed has little or no influence on the parameter estimates, is precisely the robustness of the estimator.

\section{B. Robust parameters estimation}

We detail in this section robust estimation procedures suitable for estimating the mean vector and scatter matrix within the class of elliptical distributions.

1) Maximum Likelihood Estimators: When the density generator $h_{m}($.$) is perfectly known, i.e. the p.d.f. of the underlying$ distribution is explicit, then the MLEs of $\boldsymbol{\mu}$ and $\boldsymbol{\Sigma}$ can be derived and they are given by:

$$
\begin{aligned}
\hat{\boldsymbol{\mu}}_{M L E} & =\frac{\sum_{i=1}^{N} \psi\left(t_{i}\right) \mathbf{x}_{i}}{\sum_{i=1}^{N} \psi\left(t_{i}\right)} \\
\hat{\boldsymbol{\Sigma}}_{M L E} & =\sum_{i=1}^{N} \psi\left(t_{i}\right)\left(\mathbf{x}_{i}-\hat{\boldsymbol{\mu}}\right)^{T}\left(\mathbf{x}_{i}-\hat{\boldsymbol{\mu}}_{M L E}\right),
\end{aligned}
$$

where $t_{i}=\left(\mathbf{x}_{i}-\hat{\boldsymbol{\mu}}_{M L E}\right)^{T} \hat{\boldsymbol{\Sigma}}_{M L E}^{-1}\left(\mathbf{x}_{i}-\hat{\boldsymbol{\mu}}_{M L E}\right)$ and $\psi(t)=-2 h_{m}^{\prime}(t) / h_{m}(t)$.

Note that the two quantities $\hat{\boldsymbol{\mu}}_{M L E}$ and $\hat{\boldsymbol{\Sigma}}_{M L E}$ appear on both sides of these equations, characterizing fixed-point equations $\hat{\boldsymbol{\mu}}_{M L E}=f\left(\hat{\boldsymbol{\mu}}_{M L E}, \hat{\boldsymbol{\Sigma}}_{M L E}\right)$ and $\hat{\boldsymbol{\Sigma}}_{M L E}=$ 
$g\left(\hat{\boldsymbol{\mu}}_{M L E}, \hat{\boldsymbol{\Sigma}}_{M L E}\right)$. Note that, due to the classical ML theory, solutions of such equations exist.

2) Fixed Point Estimators: Generalizing these MLEs leads to the class of $M$-estimators, introduced in hyperspectral community in [27]. More precisely, the main idea is to define a class of estimates that are not directly related to the underlying p.d.f.: $\psi($.$) is not anymore a function of h_{m}($.$) . However, the$ asymptotical distribution of these $M$-estimates are very close to those obtained in the Gaussian context, i.e. for $\hat{\boldsymbol{\Sigma}}_{S C M}$ and $\hat{\boldsymbol{\mu}}_{S M V}$. In the elliptically distributed background context, they appear to be more appropriate and robust to potential outliers present in the data. For consequence, this implies that these $M$-estimates can replace conventional Gaussian estimates in all detection schemes without degrading their performance in Gaussian context but with enhancing their performance in nonGaussian context. Among the large class of $M$-estimators, the Fixed Point (FP) estimators, according to the definition proposed by Tyler in [13], appears to be the most robust estimates satisfying the following implicit equations:

$$
\begin{gathered}
\hat{\boldsymbol{\mu}}_{F P}=\frac{\sum_{i=1}^{N} \frac{\mathbf{x}_{i}}{\left(\left(\mathbf{x}_{i}-\hat{\boldsymbol{\mu}}_{F P}\right)^{T} \hat{\boldsymbol{\Sigma}}_{F P}^{-1}\left(\mathbf{x}_{i}-\hat{\boldsymbol{\mu}}_{F P}\right)\right)^{1 / 2}}}{\sum_{i=1}^{N} \frac{1}{\left(\left(\mathbf{x}_{i}-\hat{\boldsymbol{\mu}}_{F P}\right)^{T} \hat{\boldsymbol{\Sigma}}_{F P}^{-1}\left(\mathbf{x}_{i}-\hat{\boldsymbol{\mu}}_{F P}\right)\right)^{1 / 2}}} \\
\hat{\boldsymbol{\Sigma}}_{F P}=\frac{m}{N} \sum_{i=1}^{N} \frac{\left(\mathbf{x}_{i}-\hat{\boldsymbol{\mu}}_{F P}\right)\left(\mathbf{x}_{i}-\hat{\boldsymbol{\mu}}_{F P}\right)^{T}}{\left(\mathbf{x}_{i}-\hat{\boldsymbol{\mu}}_{F P}\right)^{T} \hat{\boldsymbol{\Sigma}}_{F P}^{-1}\left(\mathbf{x}_{i}-\hat{\boldsymbol{\mu}}_{F P}\right)} .
\end{gathered}
$$

The joint solutions can be obtained using the recursive algorithm given by:

$$
\begin{gathered}
\hat{\boldsymbol{\mu}}_{F P}^{(0)}=\hat{\boldsymbol{\mu}}_{S M V} \quad \hat{\boldsymbol{\Sigma}}_{F P}^{(0)}=\hat{\boldsymbol{\Sigma}}_{S C M} \\
\left\{\begin{array}{c}
\hat{\boldsymbol{\mu}}_{F P}^{(n+1)}=f\left(\hat{\boldsymbol{\mu}}_{F P}^{(n)}, \hat{\boldsymbol{\Sigma}}_{F P}^{(n)}\right) \\
\hat{\boldsymbol{\Sigma}}_{F P}^{(n+1)}=g\left(\hat{\boldsymbol{\mu}}_{F P}^{(n)}, \hat{\boldsymbol{\Sigma}}_{F P}^{(n)}\right)
\end{array} .\right.
\end{gathered}
$$

The FP estimates have been widely investigated in statistics and signal processing literature. We refer to [28] for a detailed performance analysis. It is worth pointing out that $\hat{\boldsymbol{\Sigma}}_{S C M}$ and $\hat{\boldsymbol{\Sigma}}_{F P}$ have the same asymptotic Gaussian distribution which differs on their second order moment by a factor $\frac{m+1}{m} N$, i.e. for $N$ sufficiently large, $\hat{\boldsymbol{\Sigma}}_{F P}$ behaves as a Wishart matrix with $\frac{m}{m+1} N$ degrees of freedom. Indeed, these estimators belong to the wider class of robust $M$-estimators [29].

3) Shrinkage estimators: We present now shrinkage methods that are suitable for high dimensional problems with small number of samples (large $m$ small $N$ ). In these "large $m$ small $N$ " problems, classical estimators suffer from a distorted eigen-structure and improved estimators are required.

A common regularization approach has been widely studied, the shrinkage-SCM approach introduced in [30], [31]. Thus, in Gaussian context the regularized SCM takes the form:

$$
\begin{aligned}
& \hat{\mathbf{M}}_{S h r-S C M}(\beta)= \\
& \frac{1-\beta}{N} \sum_{i=1}^{N}\left(\mathbf{x}_{i}-\hat{\boldsymbol{\mu}}_{S M V}\right)\left(\mathbf{x}_{i}-\hat{\boldsymbol{\mu}}_{S M V}\right)^{T}+\beta \mathbf{I}_{m} .
\end{aligned}
$$

In presence of non-Gaussian, impulsive background the estimate in Eq. (26) suffers from the same drawbacks than the SCM and the class of robust estimates are more appropriate. Yet, the FP estimators described above exhibit important shortcomings in high dimensional context and they can not be computed for the undersampling case when $m>N$.

Morover, we extend here FP covariance matrix estimator to the high dimensional setting using shrinkage regularization. Let us consider now the shrinkage FP introduced in [32] and defined as the solution of the following fixed point equation:

$$
\begin{aligned}
& \hat{\mathbf{M}}_{S h r-F P}(\beta)=(1-\beta) \frac{m}{N} \times \\
& \sum_{i=1}^{N} \frac{\left(\mathbf{x}_{i}-\hat{\boldsymbol{\mu}}_{F P}\right)\left(\mathbf{x}_{i}-\hat{\boldsymbol{\mu}}_{F P}\right)^{T}}{\left(\mathbf{x}_{i}-\hat{\boldsymbol{\mu}}_{F P}\right)^{T} \hat{\mathbf{M}}_{S h r-F P}^{-1}(\beta)\left(\mathbf{x}_{i}-\hat{\boldsymbol{\mu}}_{F P}\right)}+\beta \mathbf{I}_{m},
\end{aligned}
$$

for $\beta=\in(0,1]$ and $\hat{\boldsymbol{\mu}}_{F P}$ given in Eq. (23).

It was shown in [32] that when $\beta$ tends to 0 , the proposed shrinkage estimator in Eq. (27) tends to the FP estimator in Eq. (24) whose inverse has its trace equal to $m$. A different approach that introduces a normalization constraint in the algorithm for the shrinkage FP estimates is found in [33]. Moreover, in [34], [35], [36], this estimator has been used within the Expected Likelihood framework. The optimization of the shrinkage parameter $\beta$ has been discussed in [37].

The basis of the proposed method are the FP estimators. However, the approach presented here could be extended to other $M$-estimators.

\section{Robust Kelly Anomaly Detector}

All the detection schemes explained in Section II are derived under Gaussian assumption. In this section, we explore the use of robust estimation methods presented above for anomaly detection. These can then be used as plug-in estimators in place of the unknown mean vector and/or of the covariance matrix in the detection scheme. This is a simple but often efficient method to obtain robust properties for signal processors derived under the Gaussian assumption.

The Kelly anomaly detector has the advantage that the mean vector and the covariance matrix are independent to each other and to the observation vector, which is not the case for the Generalized Kelly or the classical RXD. This allows replacing the unknown parameters by a robust FP estimators or Shrinkage estimators and the detector can be written as:

$$
\Lambda_{\text {KellyAD } \hat{\boldsymbol{\Sigma}}, \hat{\boldsymbol{\mu}}}=\left(\mathbf{x}-\hat{\boldsymbol{\mu}}_{\text {Robust }}\right)^{T} \hat{\boldsymbol{\Sigma}}_{\text {Robust }}^{-1}\left(\mathbf{x}-\hat{\boldsymbol{\mu}}_{\text {Robust }}\right) \underset{\mathcal{H}_{0}}{\stackrel{\mathcal{H}_{1}}{\gtrless}} \lambda,
$$

where $\hat{\boldsymbol{\mu}}_{\text {Robust }}$ and $\hat{\boldsymbol{\Sigma}}_{\text {Robust }}$ are those described in Section III-B. It is important to highlight that the distribution of this detector is still an open question, as far as the authors are aware. In fact, it will surely depend on the underlying particular CE distribution, i.e. the distribution will change with the choice of $h_{m}(\cdot)$.

\section{Simulations}

In this section, we validate the theoretical analysis on simulated data. Firstly, we validate through Monte-Carlo simulations the distribution of $\Lambda_{\text {Kelly } A D}$ detailed above. The 


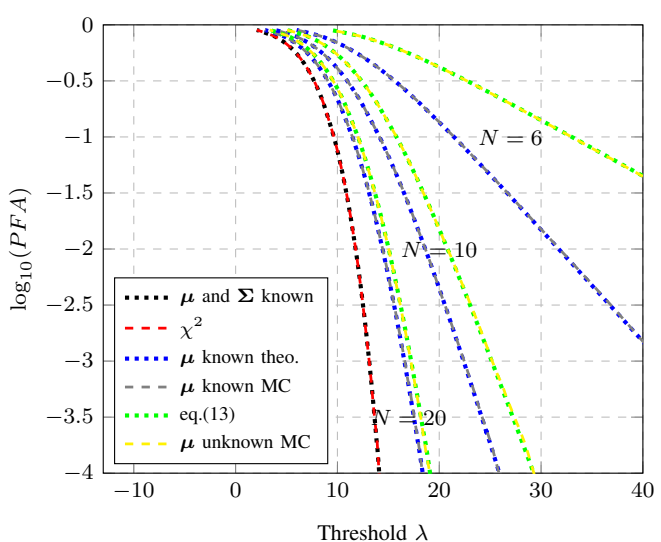

Fig. 1: PFA versus threshold for the $\Lambda_{\text {Kelly AD }}(m=5)$ when (1) $\boldsymbol{\mu}$ and $\boldsymbol{\Sigma}$ are known (Mahalanobis) (red and black curves) (2) only $\boldsymbol{\mu}$ is known (gray and blue curves) (3) Proposition II.1: both $\boldsymbol{\mu}$ and $\boldsymbol{\Sigma}$ are unknown (yellow and green curves).

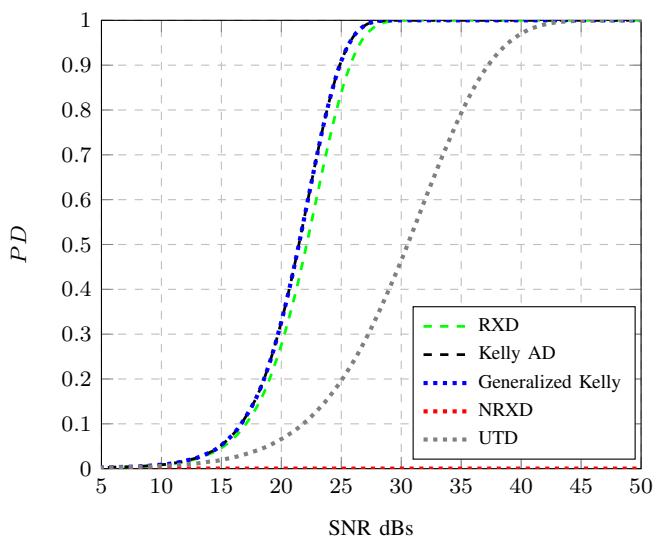

Fig. 2: Probability of detection for different SNR values, $m=$ 5, $N=10$ and $P F A=10^{-3}$ in Gaussian case.

experiments have been conducted on Gaussian vectors of dimension $m=5$ and for different values of $N$. The computations have been made through $10^{6}$ Monte-Carlo trials. The true covariance is chosen as a Toeplitz matrix whose entries are $\Sigma_{i, j}=\rho^{|i-j|}$ and where $\rho=0.4$. The mean vector is arbitrary set to have all entries equal to 3 . Exceedance plot shows the fraction of points in the data set whose Mahalanobis distance is larger than the indicated value. This is essentially a cumulative histogram of Mahalanobis distance values which correspond to the "PFA-threshold" relationship. Remark that the definition of false alarms is not unique and it depends on the application. Thus, we will rather refer to the distribution of the detector in target absent hypothesis.

Fig. 1 illustrates the distribution of the detector under null hypothesis. The case where both covariance matrix and mean vector are perfectly known corresponds to the $\chi^{2}$-distribution and the adaptive versions of the quadratic form become a $T^{2}$ Hotelling. The perfect agreement of the green and yellow curves bears out the results of Proposition II.1. Furthermore, we compare, in Fig. 2, the five proposed anomaly detectors in terms of PD for different values of the Signal-to-Noise Ratio (SNR). The experiments were on Gaussian vectors of dimen-

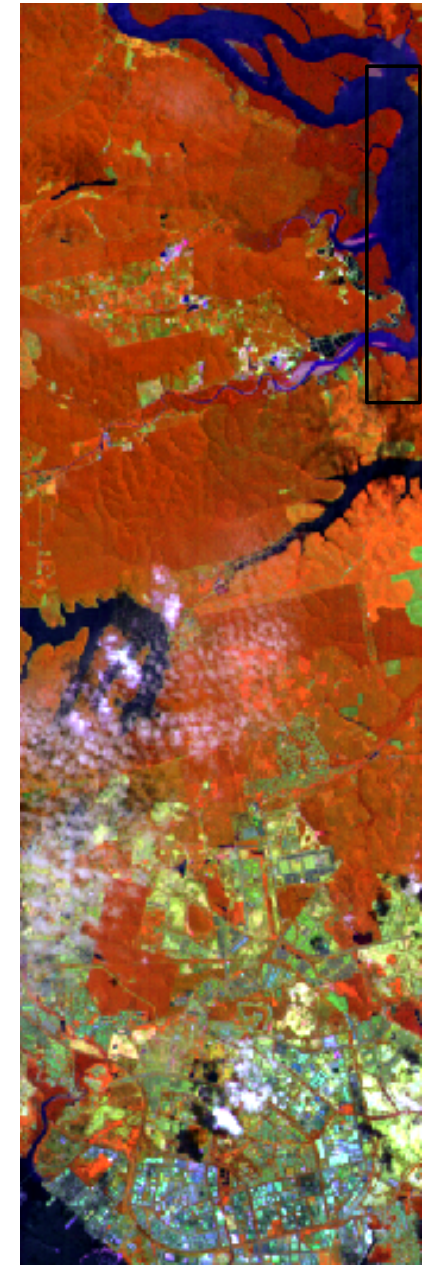

Fig. 3: True color composition of the Hyperion scene.

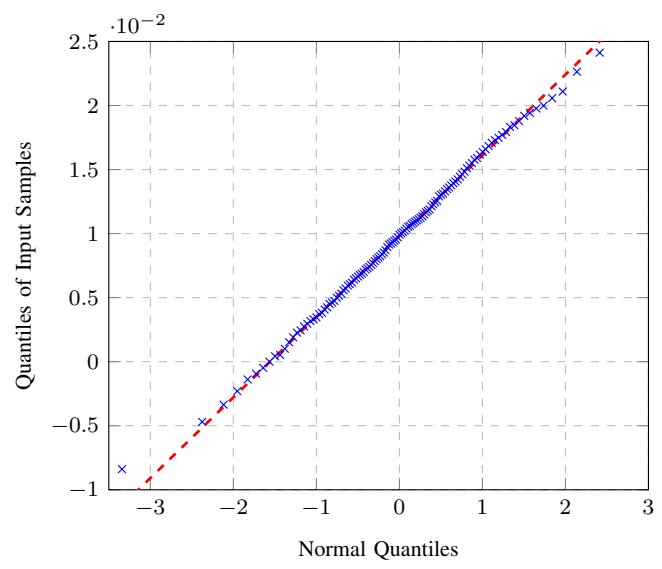

Fig. 4: Q-Q Plot of the data sample versus the Normal theoretical distribution. 


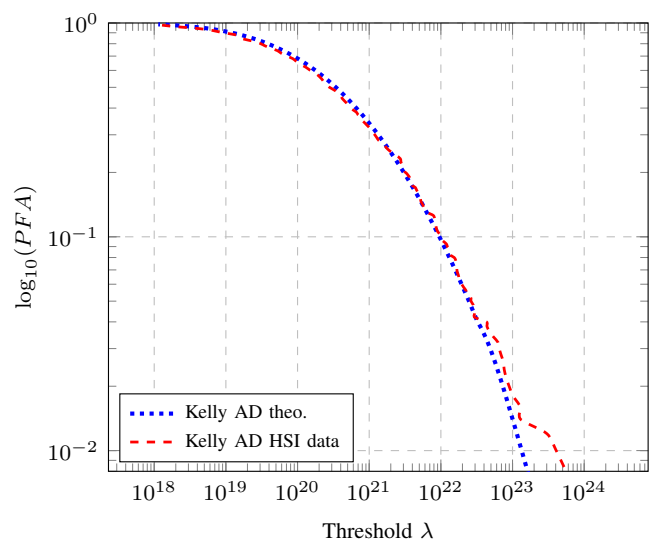

Fig. 5: Kelly AD complementary CDF of the Mahalanobis distance for a real HSI image $(m=6, N=24)$.

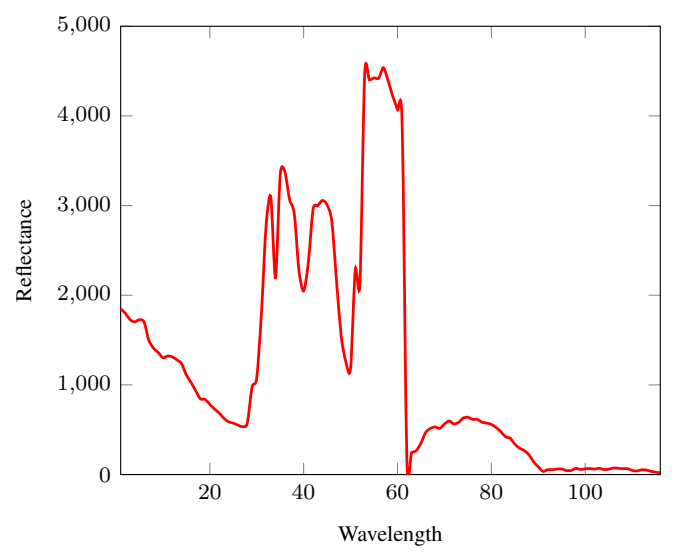

Fig. 6: Endmember used in the experiment which corresponds to land materials.

sion $m=5$, for $N=10$ and the artificial targets signature used for the simulations is the unity vector $\mathbf{p}=[1, \ldots, 1]^{T}$. On a first step, the threshold is determined to ensure exactly the same $P F A=10^{-3}$ for all detectors. The best results are obtained for the Mahalanobis-based detectors, i.e. the classical RXD, Kelly AD and generalized Kelly AD. The two detectors derived according to Kelly's approach perform fundamentally the same and slightly better than the RXD. This improvement may be due to the fact that $N+1$ data are available for the Kelly's strategies, while only $N$ samples are used in the classical RXD. The matched filter based detectors deliver poor performance in the case of the UTD, as the matching signal is the unity vector, which is shown to be not optimal even in the case the artificial targets signature used for the simulations is the unity vector. The N-RXD presents an almost flat curve as the normalization factor grows as the SNR gets higher. The outcome of this detector can be assimilated to the residual background level and its use in Gaussian environment should be avoided.

\section{REAL HYPERSPECTRAL DATA}

\section{A. Gaussian Background}

The same experiments that in simulations have been conducted on a real hyperspectral image. The scene analyzed is the NASA Hyperion sensor dataset displayed in Fig. 3. The image is constituted of $798 \times 253$ pixels and 116 spectral bands after water absorption bands have been removed. The analysis has been done on a homogenous part of the image corresponding to the water region on the top left of the image. The part extracted consists on $60 \times 20$ pixels. In order to ensure the validity of the proposed methods, we show in Fig. 4 the outcome of a classical Gaussianity test "Q-Q plot" for the selected region over the band 42. Even if this allows to "validate" the Gaussianity of each band, it cannot ensure the Gaussianity of the corresponding multivariate vector.

To avoid the well-known problem due to high dimensionality, we have chosen sequentially $m=6$ bands. In this approach, both covariance matrix and mean vector are estimated using a sliding window of size $5 \times 5$, having $N=24$ secondary data.

Fig. 5 shows the distribution of the $\Lambda_{\text {KellyAD } \hat{\Sigma}, \hat{\boldsymbol{\mu}}}$ in real hyperspectral data (red curve). We also plot the theoretical relationship defined by Eq. (13). The results obtained on real HSI data on a Gaussian distributed region agree with the theoretical relationships presented above.

Finally, we illustrate the detection capability of the proposed methods when artificial anomalies with known spectral signature are inserted on the real hyperspectral image. For this purpose, we extract the spectral signature from ground materials in Fig. 3 and the anomaly spectra is depicted in Fig. 6. Fig. 7(a) details the position and the shape of the targets. For the same fixed value of FA $P F A=0.1$, we present in Fig. 7 the outcome of the different conventional detectors for $m=6$ and $N=24$ secondary data. Note that the edges of the image are not processed and the detection maps are trimmed in function of the window size. The detectors based on the Mahalanobis distance deliver best results for detection purposes as expected and the matched filter based detectors do not detect properly the artificial targets. These detection maps are in agreement with the SNR figure detailed above. Remark that the two-pixel targets are not detected by any of the detection schemes. This problem is due to the presence of a strong target in the secondary data that pollutes the covariance matrix estimation. Its occurrence has a significant impact on the detection process and it degrades the performances of conventional detectors.

Fig. 8 shows the results of the extended $\Lambda_{\text {Kelly } A D}$ detector ( $m=6, N=24$ ) built now with FP estimators and with Shrinkage estimators, both SCM and FP. The same value of FA PFA $=10^{-1}$ is considered for the three detectors. Remark that all the anomalies of interest are now detected even those bigger than one pixel. Thereafter, due to their robustness, the proposed estimation methods allow for better detection results in Gaussian case.

Receiver Operating Characteristics (ROC) curves are widely used in signal processing to evaluate the performances of the detectors [38]. ROC curves depict the outcome for a set of thresholds instead of showing the misclassification for only one. The $x$-axis represents the PFA and the $y$-axis, the probability of detection (PD). A good detector presents high PD values at low PFA, i.e., the curve is closer to the upper 


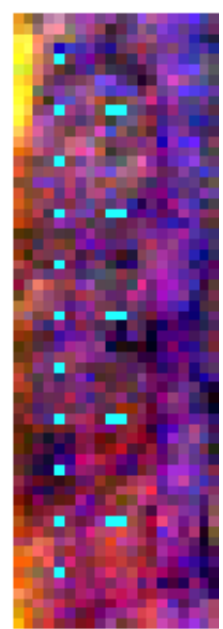

(a) Original

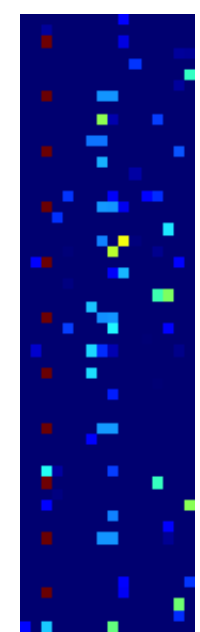

(b) RXD

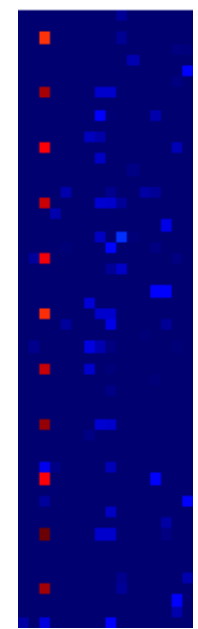

(c) Kelly AD

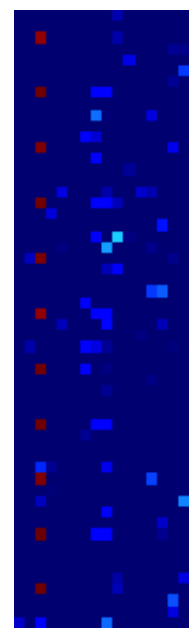

(d) G-Kelly

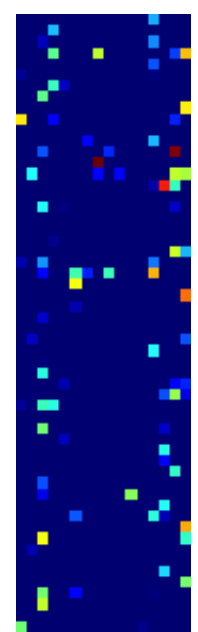

(e) N-RXD

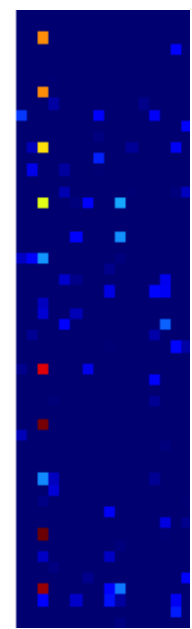

(f) UTD

Fig. 7: Conventional Anomaly Detection for artificial targets in real HSI $\left(m=6, N=24\right.$, same PFA $\left.=10^{-1}\right)$.

left corner. Fig. 9 shows the results of the ROC curves for the different detectors presented above $(m=6, N=24)$ computed on the Gaussian water region of the Hyperion image. For a fixed value of the SNR $=10 \mathrm{~dB}, \mathrm{RXD}$, Kelly AD built with the different estimators and Generalized Kelly AD exhibit perfect classification while N-RXD and UTD provide worse results.

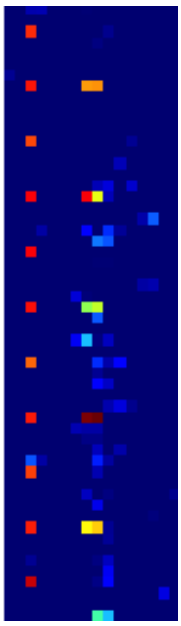

(a) FP

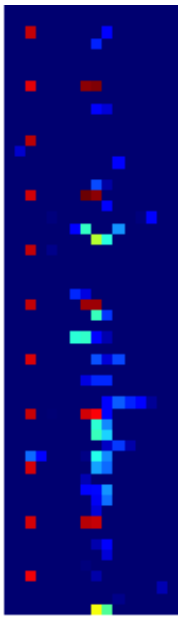

(b) Shr-SCM

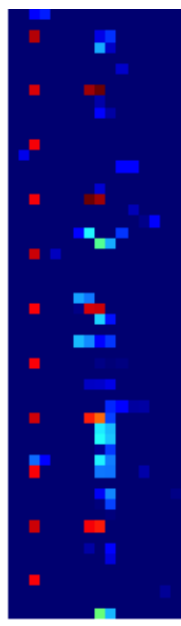

(c) Shr-FP
Fig. 8: Extended Kelly AD detectors built with robust estimates for artificial targets in real HSI $(m=6, N=24$, same PFA $=10^{-1}$ ).

\section{B. Non-Gaussian Background}

Let us now present some results on a real hyperspectral image in which the background can not be characterized with Gaussian distribution and artificial targets were introduced as anomalies. The original data set consists on $50 \times 50$ pixels with 126 bands, from which we have chosen sequentially $m=$ 9 bands, see Fig. 10 (a). For this example, both covariance

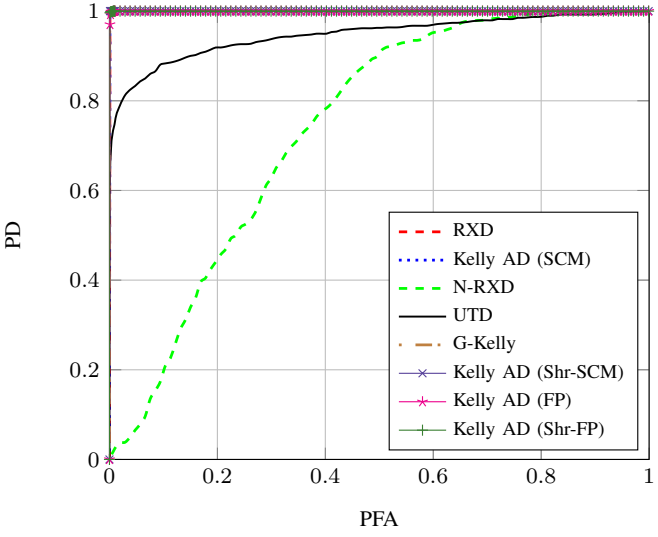

Fig. 9: ROC curve comparing the different detectors in Gaussian environment $(m=6, N=24$ and $\mathrm{SNR}=10 \mathrm{~dB})$.

matrix and mean vector are estimated using a sliding window of size $9 \times 9$ having $N=80$ secondary data. The results for the $\Lambda_{\text {Kelly } A D}$ built with classical SMV-SCM estimates, FP estimates and shrinkage estimators are shown in Fig 10, the FA being fixed at the same value of $P F A=0.03$. In this case, FP estimators and notably shrinkage FP estimates are capable of locating all the artificial targets and exhibit a lower number of false alarms. This improvement is due to the fact that FP estimates treat the outliers and impulsive samples in order for them to have a smaller contribution to the background characterization process, while the SMV-SCM estimates (and its respective shrinkage version) suffer from the presence of strong reflectance pixels in the secondary data. Remark that the shrinkage FP estimates lead to a better detection compared to FP estimates.

Let us now consider the dataset in Fig. 10(a) with all 126 bands available. In high-dimensionality problems, the SMV-SCM and the FP estimators suffer from distorted eigen-structure. This fact motivates the use of shrinkage estimators. As the background is shown to be non-Gaussian, 


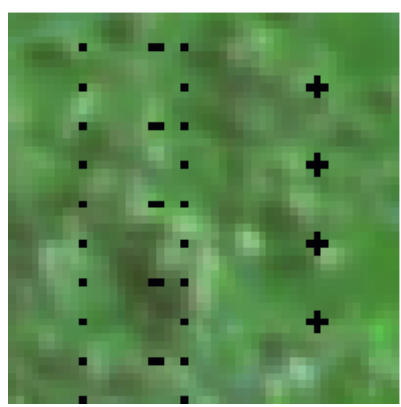

(a) Original

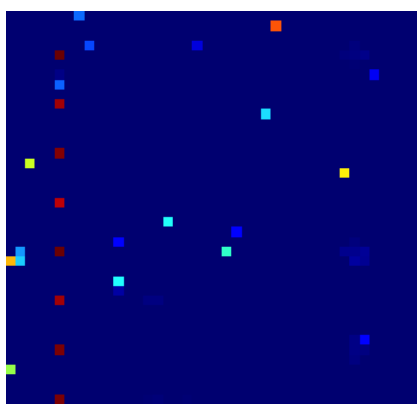

(b) SCM

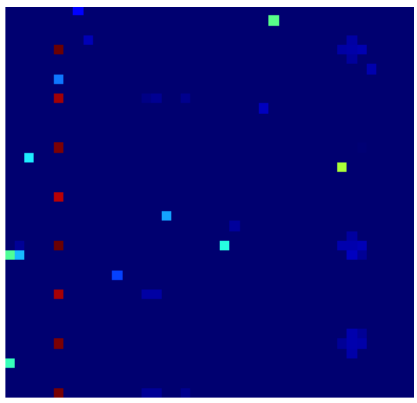

(c) Shr-SCM

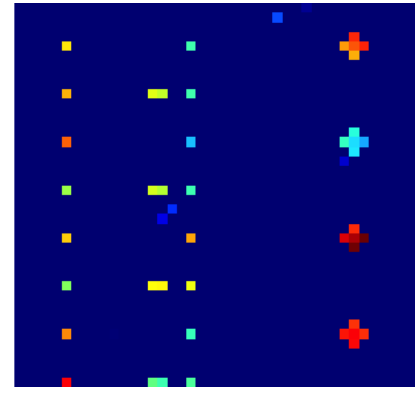

(a) FP

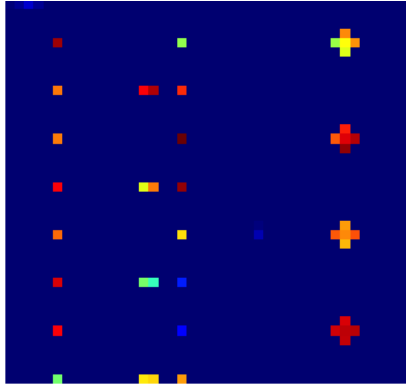

(b) Shr-FP

Fig. 10: Extended Kelly AD built with conventional and robust estimates for artificial targets in real HSI with all the bands $(m=9, N=80$, same PFA $=0.03)$.

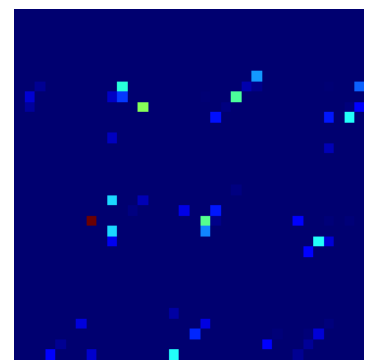

(b) SCM

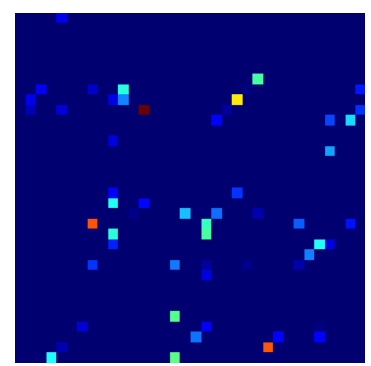

(c) Shr-SCM

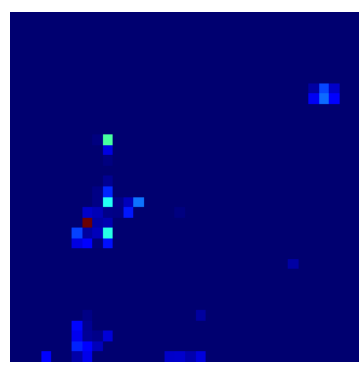

(a) FP

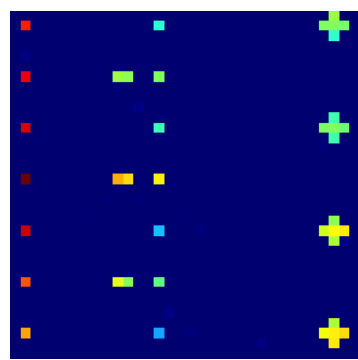

(b) Shr-FP

Fig. 11: Extended Kelly AD built with conventional and robust estimates for artificial targets in real HSI $(m=126, N=288$, same $\mathrm{PFA}=10^{-1}$ ).

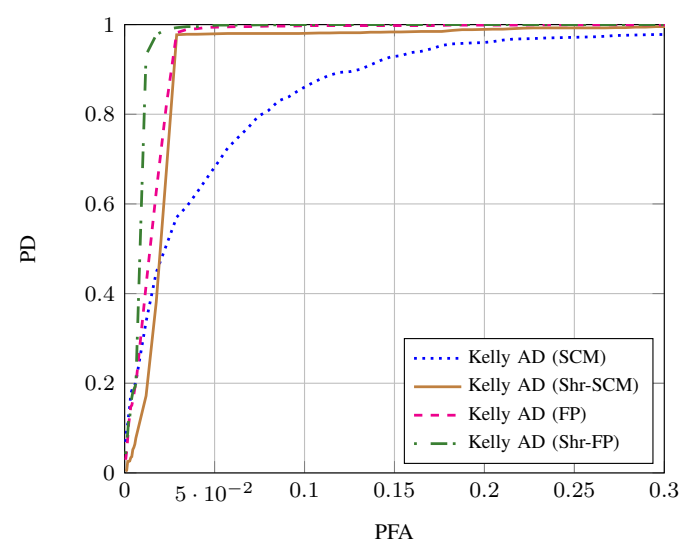

Fig. 12: ROC curve comparing the different estimation methods in non-Gaussian environment $(m=9, N=80$ and $\mathrm{SNR}=10 \mathrm{~dB}$ ). the shrinkage FP estimators are the most appropriate solutions when dealing with all the bands. We show in the Fig. 11 the results when using all the bands, $m=126$, and the sliding window has been increased to $17 \times 17, N=288$. Note that the edges of the image are not processed and the detection maps are trimmed in function of the window size. The shrinkage FP are still capable of detecting all the targets while all the other estimation techniques lead to poor detection results.

Fig. 12 displays the different ROC curves in non-Gaussian background for the image in Fig. 10 (a), with $m=9, N=80$ and a fixed value of SNR $=10 \mathrm{~dB}$. Classical SMV-SCM provide worse results than their shrinkage counterpart. Moreover, in non-Gaussian case, FP estimators and the corresponding shrinkage FP estimators allow for better detection while keeping the false alarm low. This is translated in ROC curves closer to the upper left corner and improved detection performances. 


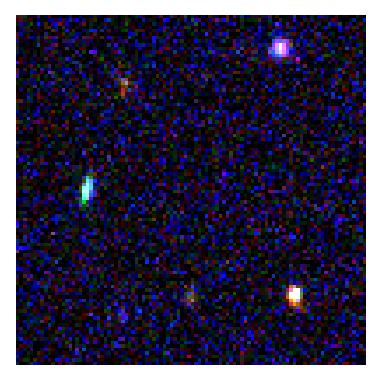

(a) MUSE data cube

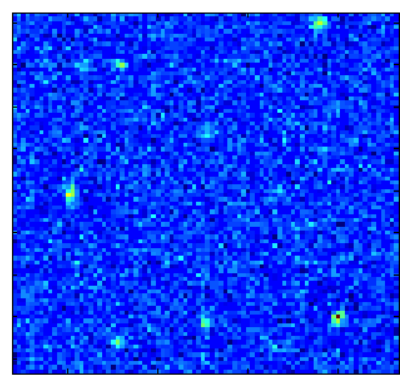

(b) SMV-SCM

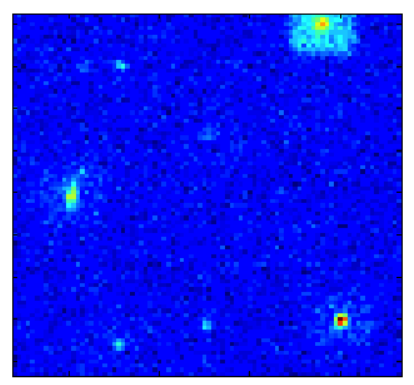

(c) FP estimates

Fig. 13: Anomaly detection in a MUSE hyperspectral image (size $300 \times 300$ ) with $m=36$ channels and $N=120$ secondary data. a) Original image. b) Kelly AD built with conventional estimates. c) Kelly AD built with FP estimates

The algorithm has also been applied for galaxy detection on the MUSE data cube. The Multi Unit Spectroscopic Explorer (MUSE) project (see [39]) aims to provide astronomers with a new generation of optical instrument, capable of simultaneously imaging the sky (in 2D) and measuring the optical spectra of the light received at a given position on the sky. MUSE was installed on the VLT telescope and operational in 2013, and its performances are expected to allow observation of far galaxies up to 100 times fainter than those presently detectable. MUSE will deliver a 3D data-cube made of a stack of images recorded at 3578 different wavelengths over the range 465-930 $\mathrm{nm}$. Each monochromatic image represents a field of view of $60 \times 60$ arcsec, recorded with a spatial sampling of 0.2 arcsec. Each record results in a data cube of size $1570 \mathrm{MB}$ encoding 3578 images of $300 \times 300$ pixels, possibly containing thousands of objects (galaxies) existing over different subsets of wavelengths.

An example of MUSE data cube image is displayed in Fig. 13 (a), from the 3578 available bands, we have chosen one band of each 100. The results for anomaly detection are presented in Fig.13 for the same imposed FA value PFA $=10^{-3}$. Note that detection with FP estimators (c) provides better and clearer results than the classical ones (b).

These examples illustrate the robust behavior of FP estimators in non-Gaussian environments or for close targets detection problems.

\section{CONCLUSION}

The classical RXD test is explored and compared to other four detectors. The different advantages and drawbacks for the different detection schemes are commented. Furthermore, the comparison is performed through Monte Carlo simulations in Gaussian context and extended to real hyperspectral data with simulated anomalies. The family of elliptical distributions is considered for impulsive background characterization in hyperspectral imaging. In this context, robust estimation methods for mean vector and covariance matrix are used to overcome the non-Gaussianity of the background and the presence of outliers or strong scatters in the secondary data. Moreover the robust methods presented in this work outperform significantly the classical Gaussian-based SMV-SCM. Therefore, robust estimators offer a versatile alternative to Gaussian estimates.
They allow to obtain better performances in impulsive environments while keeping good results in Gaussian background. The theoretical improvement provided by the robustness of the estimators is borne out through two real hyperspectral images.

\section{REFERENCES}

[1] D. Manolakis and G. Shaw, "Detection algorithms for hyperspectral imaging applications," Signal Processing Magazine, IEEE, vol. 19, no. 1, pp. 29-43, 2002.

[2] D. Manolakis, E. Truslow, M. Pieper, T. Cooley, and M. Brueggeman, "Detection algorithms in hyperspectral imaging systems: An overview of practical algorithms," Signal Processing Magazine, vol. 31, no. 1, pp. 24-33, 2014.

[3] M. T. Eismann, A. D. Stocker, and N. M. Nasrabadi, "Automated hyperspectral cueing for civilian search and rescue," Proceedings of the IEEE, vol. 97, no. 6, pp. 1031-1055, 2009.

[4] C.-I. Chang and S.-S. Chiang, "Anomaly detection and classification for hyperspectral imagery," Geoscience and Remote Sensing, IEEE Transactions on, vol. 40, no. 6, pp. 1314-1325, 2002.

[5] S. Matteoli, M. Diani, and G. Corsini, "A tutorial overview of anomaly detection in hyperspectral images," Aerospace and Electronic Systems Magazine, vol. 25, no. 7, pp. 5 -28, july 2010.

[6] N. Nasrabadi, "Hyperspectral target detection: An overview of current and future challenges," Signal Processing Magazine, IEEE, vol. 31, no. 1, pp. 34-44, 2014.

[7] D. W. Stein, S. G. Beaven, L. E. Hoff, E. M. Winter, A. P. Schaum, and A. D. Stocker, "Anomaly detection from hyperspectral imagery," Signal Processing Magazine, IEEE, vol. 19, no. 1, pp. 58-69, 2002.

[8] S. Matteoli, M. Diani, and J. Theiler, "An overview of background modeling for detection of targets and anomalies in hyperspectral remotely sensed imagery," Journal of Selected Topics in Applied Earth Observations and Remote Sensing, vol. 7, no. 6, pp. 2317-2336, 2014.

[9] D. Manolakis and D. Marden, "Non gaussian models for hyperspectral algorithm design and assessment," in Geoscience and Remote Sensing Symposium, (IGARSS), 2002 IEEE International, vol. 3. IEEE, 2002, pp. 1664-1666.

[10] J. Theiler, C. Scovel, B. Wohlberg, and B. R. Foy, "Elliptically contoured distributions for anomalous change detection in hyperspectral imagery," Geoscience and Remote Sensing Letters, IEEE, vol. 7, no. 2, pp. 271275, 2010.

[11] D. Kelker, "Distribution theory of spherical distributions and a locationscale parameter generalization," Sankhyā: The Indian Journal of Statistics, Series A, pp. 419-430, 1970.

[12] K.-T. Fang, S. Kotz, and K. W. Ng, Symmetric multivariate and related distributions. Chapman and Hall, 1990.

[13] D. Tyler, "A distribution-free $m$-estimator of multivariate scatter," The Annals of Statistics, vol. 15, no. 1, pp. 234-251, 1987.

[14] F. Gini and M. V. Greco, "Covariance matrix estimation for CFAR detection in correlated heavy tailed clutter," Signal Processing, vol. 82, no. 12, pp. 1847-1859, December 2002.

[15] J. Frontera-Pons, M. Mahot, J. Ovarlez, F. Pascal, S. Pang, and J. Chanussot, "A class of robust estimates for detection in hyperspectral images using elliptical distributions background," in Geoscience and Remote Sensing Symposium (IGARSS), 2012 IEEE International. IEEE, 2012, pp. 4166-4169. 
[16] J. Frontera-Pons, M. A. Veganzones, S. Velasco-Forero, F. Pascal, J.-P. Ovarlez, J. Chanussot et al., "Robust anomaly detection in hyperspectral imaging," in Geoscience and Remote Sensing Symposium (IGARSS), 2014 IEEE International, 2014.

[17] A. K. Gupta and D. K. Nagar, Matrix Variate Distributions. Chapman \& Hall/CRC, 2000.

[18] I. Reed and X. Yu, "Adaptive multiple-band cfar detection of an optical pattern with unknown spectral distribution," Acoustics, Speech and Signal Processing, IEEE Transactions on, vol. 38, no. 10, pp. 17601770, 1990.

[19] E. J. Kelly, "An adaptive detection algorithm," Aerospace and Electronic Systems, IEEE Transactions on, no. 2, pp. 115-127, 1986.

[20] P. C. Mahalanobis, "On the generalized distance in statistics," Proceedings of the National Institute of Sciences (Calcutta), vol. 2, pp. 49-55, 1936.

[21] M. Bilodeau and D. Brenner, Theory of multivariate statistics. New York: Springer, 1999.

[22] E. W. Weisstein, CRC concise encyclopedia of mathematics. CRC press, 2010.

[23] T. W. Anderson, An Introduction to Multivariate Statistical Analysis. John Wiley \& Sons, New York, 1984.

[24] J. C. Harsanyi, "Detection and classification of subpixel spectral signatures in hyperspectral image sequences," Ph.D. dissertation, University of Maryland Baltimore County, 1993.

[25] J. Frontera-Pons, F. Pascal, and J.-P. Ovarlez, "Adaptive non-zero mean gaussian detection and application to hyperspectral imaging," arXiv preprint arXiv:1404.2977, 2014.

[26] E. Ollila, D. E. Tyler, V. Koivunen, and H. V. Poor, "Complex elliptically symmetric distributions: survey, new results and applications," Signal Processing, IEEE Transactions on, vol. 60, no. 11, pp. 5597-5625, 2012.

[27] J. Ovarlez, S. Pang, F. Pascal, V. Achard, and T. Ng, "Robust detection using the sirv background modelling for hyperspectral imaging," in Geoscience and Remote Sensing Symposium (IGARSS), 2011 IEEE International. IEEE, 2011, pp. 4316-4319.

[28] F. Pascal, P. Forster, J.-P. Ovarlez, and P. Larzabal, "Performance analysis of covariance matrix estimates in impulsive noise," Signal Processing, IEEE Transactions on, vol. 56, no. 6, pp. 2206-2217, June 2008.

[29] P. J. Huber, "Robust estimation of a location parameter," The Annals of Mathematical Statistics, vol. 35, no. 1, pp. 73-101, 1964.

[30] Y. Abramovich, "Controlled method for adaptive optimization of filters using the criterion of maximum SNR," Radio Eng. Electron. Phys, vol. 26, no. 3, pp. 87-95, 1981.

[31] B. D. Carlson, "Covariance matrix estimation errors and diagonal loading in adaptive arrays," Aerospace and Electronic Systems, IEEE Transactions on, vol. 24, no. 4, pp. 397-401, 1988.

[32] F. Pascal, Y. Chitour, and Y. Quek, "Generalized robust shrinkage estimator and its application to stap detection problem," arXiv preprint arXiv:1311.6567, 2013.

[33] Y. Chen, A. Wiesel, and A. O. Hero, "Robust shrinkage estimation of high-dimensional covariance matrices," Signal Processing, IEEE Transactions on, vol. 59, no. 9, pp. 4097-4107, 2011.

[34] Y. I. Abramovich and O. Besson, "Covariance matrix estimation in complex elliptic distributions using the expected likelihood approach," in Acoustics, Speech and Signal Processing (ICASSP), 2013 IEEE International Conference on. IEEE, 2013, pp. 6476-6480.

[35] Y. Abramovich and O. Besson, "Regularized covariance matrix estimation in complex elliptically symmetric distributions using the expected likelihood approach-part 1: The over-sampled case," Signal Processing, IEEE Transactions on, vol. 61, no. 23, pp. 5807-5818, 2013.

[36] O. Besson and Y. Abramovich, "Regularized covariance matrix estimation in complex elliptically symmetric distributions using the expected likelihood approach-part 2: The under-sampled case," Signal Processing, IEEE Transactions on, vol. 61, no. 23, pp. 5819-5829, 2013.

[37] R. Couillet and M. R. McKay, "Large dimensional analysis and optimization of robust shrinkage covariance matrix estimators," Journal of Multivariate Analysis, vol. 131, pp. 99-120, 2014.

[38] J. P. Egan, "Signal detection theory and $\{$ ROC $\}$ analysis," 1975.

[39] "Official website of the muse project "http://muse.univ-lyon1.fr/"." [Online]. Available: "http://muse.univ-lyon1.fr/" 